\title{
Nucleus accumbens is involved in human action monitoring: evidence from invasive electrophysiological recordings
}

\author{
Thomas F. Münte ${ }^{1, *}$, Marcus Heldmann², Hermann Hinrichs' ${ }^{2}$, Josep Marco-Pallares ${ }^{1}$, \\ Ulrike M. Krämer ${ }^{1}$, Volker Sturm ${ }^{3}$ and Hans-Jochen Heinze ${ }^{2}$ \\ 1. Department of Neuropsychology, University of Magdeburg, Magdeburg, Germany \\ 2. Department of Neurology II, University of Magdeburg, Magdeburg, Germany \\ 3. Department of Stereotactic and Functional Neurosurgery, University of Cologne, Cologne, Germany \\ Edited by: Robert T. Knight, University of California Berkeley, USA \\ Reviewed by: Maurizio Corbetta, Washington University, USA \\ George R. Mangun, University of California at Davis, USA
}

\begin{abstract}
The Nucleus accumbens (Nacc) has been proposed to act as a limbic-motor interface. Here, using invasive intraoperative recordings in an awake patient suffering from obsessive-compulsive disease (OCD), we demonstrate that its activity is modulated by the quality of performance of the subject in a choice reaction time task designed to tap action monitoring processes. Action monitoring, that is, error detection and correction, is thought to be supported by a system involving the dopaminergic midbrain, the basal ganglia, and the medial prefrontal cortex. In surface electrophysiological recordings, action monitoring is indexed by an error-related negativity (ERN) appearing time-locked to the erroneous responses and emanating from the medial frontal cortex. In preoperative scalp recordings the patient's ERN was found to be significantly increased compared to a large $(n=83)$ normal sample, suggesting enhanced action monitoring processes. Intraoperatively, error-related modulations were obtained from the Nacc but not from a site $5 \mathrm{~mm}$ above. Importantly, crosscorrelation analysis showed that error-related activity in the Nacc preceded surface activity by 40 ms. We propose that the Nacc is involved in action monitoring, possibly by using error signals from the dopaminergic midbrain to adjust the relative impact of limbic and prefrontal inputs on frontal control systems in order to optimize goal-directed behavior.
\end{abstract}

Keywords: action monitoring, nucleus accumbens, event-related brain potentials, intracranial recordings, neurosurgery

\section{INTRODUCTION}

We continuously evaluate our actions in order to detect and correct performance errors suggesting that we are endowed with a cognitive system that monitors and optimizes our behavior. Recordings of event-related brain potentials (ERPs) from the intact scalp have provided neurophysiological evidence for the existence of such a system in the human: An erroneous button press in a performance task elicits the "error-related" negativity (ERN or $\mathrm{Ne}$ ) having a peak latency around $60 \mathrm{~ms}$ and a medial frontal scalp distribution. The ERN is believed to be generated in the anterior cingulate cortex (Falkenstein et al., 1990; Gehring et al., 1993; Luu and Tucker, 2001; Rodriguez-Fornells et al., 2002; Rollnik et al., 2004; Ullsperger and von Cramon, 2003). Electrophysiological findings have been supported by functional neuroimaging results that corroborate a role of the medial prefrontal cortex (anterior cingulate cortex and adjacent pre-SMA) in the processing of performance errors (Botvinick et al., 2001; Carter et al., 1998; Fassbender et al., 2004; Hester et al., 2004; Ullsperger and von Cramon, 2001, 2003).

*Correspondence: Thomas F. Münte, Department of Neuropsychology, University of Magdeburg, Gebäude 24, Universitätsplatz 2, 39106 Magdeburg, Germany. e-mail: thomas.muente@medizin.uni-magdeburg.de

Received: 27 September 2007; paper pending published: 29 November 2007; accepted: 03 January 2008; published online: 28 March 2008.

Citation: Front. Hum. Neurosci. (2008) 1: 11. doi: 10.3389/neuro.09.011.2007

Copyright (ㄷ 2008 Münte, Heldmann, Hinrichs, Marco-Pallares, Krämer, Sturm and Heinze. This is an open-access article subject to an exclusive license agreement between the authors and the Frontiers Research Foundation, which permits unrestricted use distribution, and reproduction in any medium, provided the original authors and source are credited.
Importantly, the ERN has been proposed to be driven by reinforcement learning signals originating in the mesencephalic dopamine system (MDS; Holroyd and Coles, 2002). Briefly, a monitoring system located in the basal ganglia is thought to evaluate internal "efference copies" of ongoing actions. From this information, the monitoring system predicts whether ongoing events will end in success or failure. When the monitoring system revises its predictions for the better, it induces a phasic increase in the activity of the MDS (see also, Barto, 1995; Houk et al., 1995; Montague et al., 1996). Conversely, if the system revises its predictions for the worse, as in performance errors, it induces a phasic decrease in the activity of the MDS. These positive and negative reward prediction error signals are carried by the MDS to various brain areas including the anterior cingulate cortex, where they are used to improve performance on the task at hand in line with principles of reinforcement learning (Schultz, 2006).

In addition to its projections to the medial prefrontal cortex the mesencephalic dopamine system also projects to the Nucleus accumbens (Nacc), which is considered to be an important motivational brain area (Kelley and Berridge, 2002) and therefore part of the brain's reward system. It has been implicated in the etiology of craving and obsession (Ikemoto and Panksepp, 1999; Redish and Johnson, 2007). We will consider the proposed functions of the Nacc in some detail in the discussion. Importantly, the Nacc has recently become the target of neurosurgical intervention (Kuhn et al., 2007; Schlaepfer et al., 2008; Sturm et al., 2003; van Kuyck et al., 2007). Similar to the approach routinely used for the subthalamic nucleus in Parkinson's disease, electrodes connected to a stimulator placed underneath the pectoral muscle are implanted in the Nacc for the relief of obsessive compulsive disorder (OCD), depression, and Tourette's syndrome. Operations may be carried out while 
the patient is fully awake and therefore able to perform psychological tasks during the procedure. Here we report invasive recordings carried out in a 39-year old male patient suffering from compulsions (checking, washing, repeating) and obsessions since age 11, who received deep brain stimulation via an electrode placed in the right Nacc. A standard form of the flanker interference task was used, which has been employed widely to study action monitoring processes in humans (Riba et al., 2005; Rodriguez-Fornells et al., 2002; Rollnik et al., 2004). The motivation for using a flanker task was two-fold. First, previous investigations using similar tasks and surface ERPs have found evidence for increased action monitoring in OCD patients (Gehring et al., 2000; Hajcak and Simons 2002; Johannes et al., 2001, 2002; Nieuwenhuis et al., 2005; Santesso et al., 2006). Second, and more importantly, because of its dopaminergic innervation we hypothesized that we should be able to detect errorrelated electrophysiological activity in the Nacc. Such a finding would have implications for how the Nacc functions as a limbic-motor interface, as error-driven dopaminergic input to the Nacc might serve to change the weights given to contextual and emotional information coming from hippocampus and amygdale, respectively, and cognitive information coming from prefrontal cortex in the selection of goal-directed behaviours (Grace, 2000).

\section{MATERIALS AND METHODS}

All procedures were approved by the ethical review boards of the University of Cologne (patient recordings) and University of Magdeburg (control participants).

\section{Patient}

A 39-year old male patient was investigated who suffered from severe and therapy-refractory OCD (ICD 10 classification: F42.2) characterized by compulsive checking of the hands, washing and repetitive behaviour, as well as excessive rumination of thoughts. Onset of the disorder was age 11. At the time of the operation he was on venlafaxine (225 mg/day), a reuptake inhibitor of serotonin and norepinephrine. Prior therapies (all unsuccessful) had included cognitive behavioural therapy, exposition therapy, supportive psychotherapy and drug treatment with clomipramine, imipramine, fluvoxamine, citalopram, and ziprasidon. He was single and a third year student of biology and geology at the time of testing. He had been employed as an office worker prior to returning to school in his late thirties. Comprehensive neuropsychological testing revealed no cognitive or memory disturbances (Computerized test battery for the assessment of attention functions, Zimmermann and Fimm, 2002; Verbaler Lern- und Merkfähigkeitstest, Helmstaedter et al., 2001, German adaptation of Rey's Auditory Verbal Learning Test, RAVLT; Lezak, 1995; among other tests).

\section{Control participants}

A total of 83 normal participants (mean age 30.7 years) with no history of neurological and psychiatric illness served as controls. Data from subsamples of the control group have been described previously (Riba et al. 2005; Rodriguez-Fornells et al., 2002; Rollnik et al., 2004).

\section{Stimuli and procedure}

The Eriksen flanker task (Eriksen and Eriksen, 1974) was used, which requires to respond to the center letter of a 5 -letter array with either a left-hand (for letter $\mathrm{H}$ ) or right-hand response (letter S). Additional letters flanking the target letter either favoured the target response (congruent trials, HHHHH or SSSSS) or primed the other response (incongruent trials, HHSHH or SSHSS). To increase the number of errors produced, $60 \%$ of the trials were incongruent. Each stimulus array subtended about $2.5^{\circ}$ of visual angle in width, and a fixation cross was presented in the middle of the computer monitor just below the target letter in the array. The duration of the stimuli was $100 \mathrm{~ms}$, and an SOA between 900 and $1500 \mathrm{~ms}$ was used. Letter/hand assignments were counterbalanced between subjects and maintained in both sessions. Prior to the first experimental session, subjects were trained with 200 trials to reach a reaction time (RT) baseline level and they were given feedback about their performance. The goal of this procedure was to aim for a reaction time that would yield approximately $10-15 \%$ of errors. The experiment proper consisted of 6 blocks of 4 minutes each containing 200 stimuli each. A 30 -second rest period was allowed between blocks. Subjects were required to respond to the stimuli as fast as possible.

Of the 83 normal subjects, about half took part in a condition in which error corrections were encouraged (i.e., the instruction was: Whenever you detect an error, you should correct it by giving the appropriate response), while others were tested with the instruction to not correct performance errors (correction forbidden condition). ERN amplitudes have been shown to be virtually identical in a within-subject comparison (Fiehler et al., 2005; Rodriguez-Fornells et al., 2002), which is why we pooled data from both conditions for the normative sample. Nonetheless, because corrective responses might interfere with posterror adaptation processes (i.e., posterror slowing), the normal sample for the behavioural comparisons was restricted to the 41 subjects who performed in a "correction forbidden" condition. Also, the patient was tested with correction forbidden instructions.

\section{Electrophysiological recording/control participants}

The electroencephalogram was recorded from the scalp from 29 standard positions (Fp1/2, F3/4, C3/4, T3/4, T5/6, P3/4, 01/2, F7/8, Fz, Cz, Pz, $\mathrm{Fc} 3 / 4, \mathrm{FT} 7 / 8, \mathrm{Cp} 3 / 4, \mathrm{TP} 7 / 8, \mathrm{P03} / 4$ ) of the International 10-10 System (Chatrian et al., 1985, 1988). Biosignals were re-referenced off-line to the mean of the activity at the two mastoid leads. Vertical eye movements were monitored with an electrode at the infraorbital ridge of the left eye. Electrode impedances were kept below $5 \mathrm{kOhm}$. The electrophysiological signals were filtered with a bandpass of $0.1-50 \mathrm{~Hz}$ and digitized at a rate of $250 \mathrm{~Hz}$. Trials on which base-to-peak electro-oculogram (EOG) amplitude exceeded $50 \mu \mathrm{V}$, amplifier saturation occurred, or the baseline shift exceeded $300 \mu \mathrm{V} / \mathrm{s}$ were automatically rejected.

Response-locked ERPs were averaged starting $200 \mathrm{~ms}$ before the response of the subject until $624 \mathrm{~ms}$ after response onset. The baseline used for the response-locked ERN was between -200 and $0 \mathrm{~ms}$. ERPs used for the figures and statistical tests were band-passed filtered 1-8 $\mathrm{Hz}$ (half amplitude down).

\section{Electrophysiological recording/patient}

On the day prior to the operation, the patient was tested using the same set-up as described for the control participants. He finished a total of 10 experimental blocks of 80 stimuli each.

During the operation, EEG signals from the scalp surface were recorded using needle-electrodes (stainless steel) placed at positions $\mathrm{Fz}$, $\mathrm{Cz}, \mathrm{F} 3, \mathrm{~F} 4$ and Pz according to the International 10-20 system (Chatrian et al., 1985, 1988). All channels were referenced to the right mastoid. In two subsequent sessions, local field potentials (LFPs) were recorded from inside the shell of the Nacc and $5 \mathrm{~mm}$ above the border of the Nacc shell (controlled by a micro drive unit equipped with "Ben Gun" assembly) using the macro electrode contact (stainless steel) at the tip of the micromacro electrode (Inomed GmbH, Germany). Signals from the macro electrode were referenced to the electrodes' guide tube. All signals were acquired at a bandwidth of $0.1-100 \mathrm{~Hz}$ and a digitization rate of $250 \mathrm{~Hz}$. The baseline for local field potentials and event related potentials was -400 and -200 ms before response.

Response-locked ERPs were obtained in the same way as described for the control group. In addition to standard analysis in the time-domain, we also examined time-frequency behaviour of the electrical activity elicited by the response (see Marco-Pallares et al., 2008, for a more extensive description). To this end, 4 second epochs were generated (2000 ms before and after the stimulus-onset). Epochs exceeding $\pm 50 \mu \mathrm{V}$ in EOG or 
EEG were removed from further analysis. Single-trial data was convoluted using a complex Morlet wavelet:

$w\left(t, f_{0}\right)=\left(2 \pi \sigma_{t}^{2}\right)^{-\frac{1}{2}} e^{\frac{-t^{2}}{2 \sigma_{t}^{2}}} e^{2 i \pi f_{0} t}$

with the relation $f_{0} / \sigma_{f}$ [where $\sigma_{f}=1 /\left(2 \pi \sigma_{t}\right)$ ] set to 6.7 (Tallon-Baudry et al., 1997). Changes in time varying energy (square of the convolution between wavelet and signal) in the studied frequencies (from 1 to $40 \mathrm{~Hz}$; linear increase) with respect to baseline ( -300 to $0 \mathrm{~ms}$ with regard to stimulus onset) were computed for each trial and averaged separately for error and correct trials.

\section{RESULTS}

\section{Behavior}

Reaction times in correct trials were $447 \mathrm{~ms}$ (SD 80) in the healthy control subjects $(n=41)$ and $434 \mathrm{~ms}$ in the patient during presurgical testing. The reaction time to error trials was $379 \mathrm{~ms}$ (SD 70) in the control participants and $436 \mathrm{~ms}$ in the patient. Control subjects committed choice errors in $11.78 \%$ (SD 6.93), while the OCD patient's errors amounted to $19.8 \%$.

To investigate posterror adaptations of behaviour, the degree of posterror slowing (Rabbitt, 1966, 1968, 2002) was determined by subtracting the reaction times to correct trials following correct trials from those to correct trials following error trials. Mean posterror slowing was $28 \mathrm{~ms}$ (SD 40) in the control sample, while the patient's posterror slowing amounted to $70.5 \mathrm{~ms}$. The estimated percentage (using the program provided by Crawford and Howell, 1998) of the control population falling below the patient's score was $85 \%$. Thus, the patient's posterror slowing was clearly in upper range of the normal sample.

During the operation, the reaction times of the patient were $391 \mathrm{~ms}$ for correct and $338 \mathrm{~ms}$ for error trials. He committed choice errors in $25.2 \%$ of the trials. Thus, the patient was able to perform the task adequately in spite of the challenges of surgery and the busy environment in the operating theatre.

\section{ERPs control participants}

Grand average ERPs time-locked to the responses are shown in Figure 1A. A clear phasic negativity emerged for the errors starting with the button press and reaching its maximum at approximately $70 \mathrm{~ms}$. This negativity is followed by a positivity (error positivity) with a maximum at about $250 \mathrm{~ms}$. Figure 1B shows the distribution of the ERN amplitudes quantified as the mean amplitude of the difference wave error - correct (time-window 0-100 ms) at electrode $\mathrm{Cz}$ (mean $-5.01 \mu \mathrm{V}$, SD 3.57).

\section{Patient pretest}

Average response-locked ERPs of the patient recorded on the day prior to the operation are shown in Figure 2. A typical ERN was observed for this patient. The topographical distribution is illustrated by the isovoltage map in Figure 2B. A frontocentral maximum was obtained. The ERN was quantified as described for the control participants in the previous section at electrode $\mathrm{Cz}(-11.82 \mu \mathrm{V})$. To determine whether the difference between the patient's ERN amplitude and that of the control participants was significant, the test described by Crawford and Howell (1998) was computed $\left(t=-1.896, p_{\text {(one-tailed) }}=0.03\right)$. The estimated percentage of the normal population having a higher amplitude than the patient was $3.07 \%$ (95\% lower/upper confidence limits on the percentage 1.17\%/6.14\%). Thus, it can be concluded that the patient has an extraordinarily large ERN amplitude.

\section{Patient intraoperative recording}

During the operation the patient completed 2 experimental blocks of 7.5 minutes each. The electrode was positioned $5 \mathrm{~mm}$ above $(-5 \mathrm{~mm}$,
A

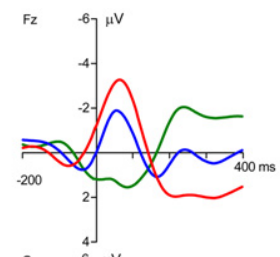

$\mathrm{Cz}$

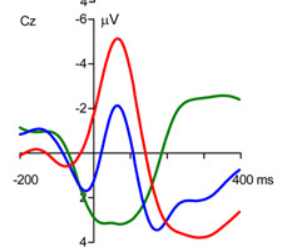

B

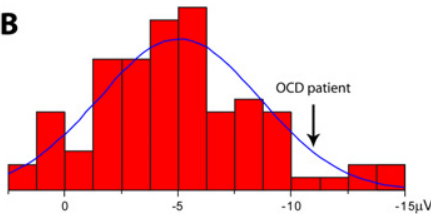

- correct
Figure 1. Event-related potentials of the normal sample. (A) Grand average ERPs of the normal participants $(n=83)$. A clear phasic negativity is seen in response to the errors starting at the time of the erroneous button press and reaching its maximum at about $70 \mathrm{~ms}$. (B) Distribution of ERN amplitudes (quantified as the mean amplitude 0-100 ms of the error - correct difference wave) in the normal sample. The amplitude of the OCD patient is marked by an arrow.
A

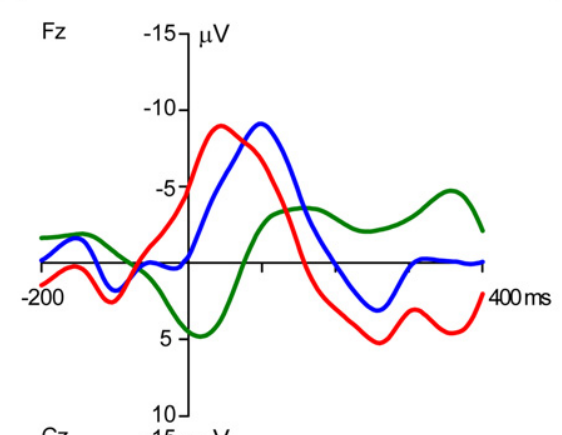

B

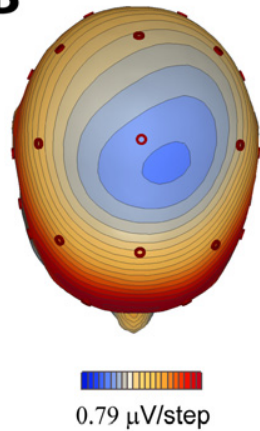

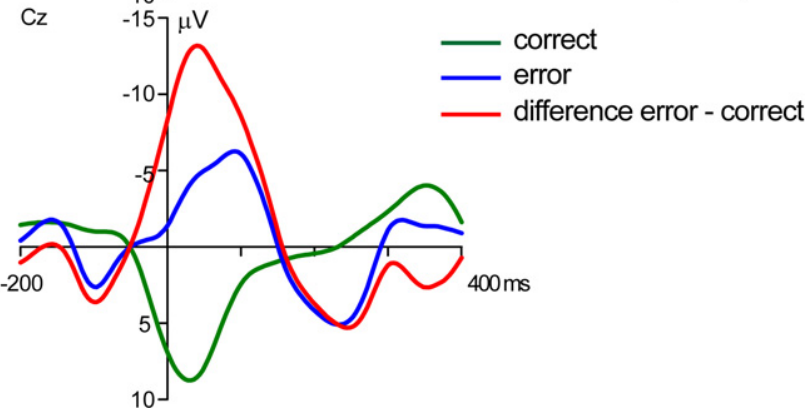

Figure 2. Event-related potentials from the OCD-patient. Pretest prior to the operation. (A) Event-related brain responses time-locked to the button press response recorded from the scalp ( $F z, C z$ sites referenced to average activity at the two mastoid processes). (B) The topographic map shows the distribution of the patient's ERN. A typical fronto-central maximum is observed.

block 1) and directly within (Nacc, block 2) the target region, i.e., the shell of the Nacc. The final position of the electrode tip can be derived from Figure 3. Figure 4 shows the surface ERPs ( $\mathrm{Cz}$ referenced to $\mathrm{A} 1$ ) together with those of the respective depth recordings. Clearly, an ERN is observed for the errors but not for correct responses. Also, for the $0 \mathrm{~mm}$ depth recording a clear error-related modulation is observed. In order to account for the fact that errors are usually associated with a faster reaction time than correct responses, we also computed an average ERP for a subset of the correct trials that were matched to the error trials in terms of their RT. Again, averaged activity from the target region showed an error-related 


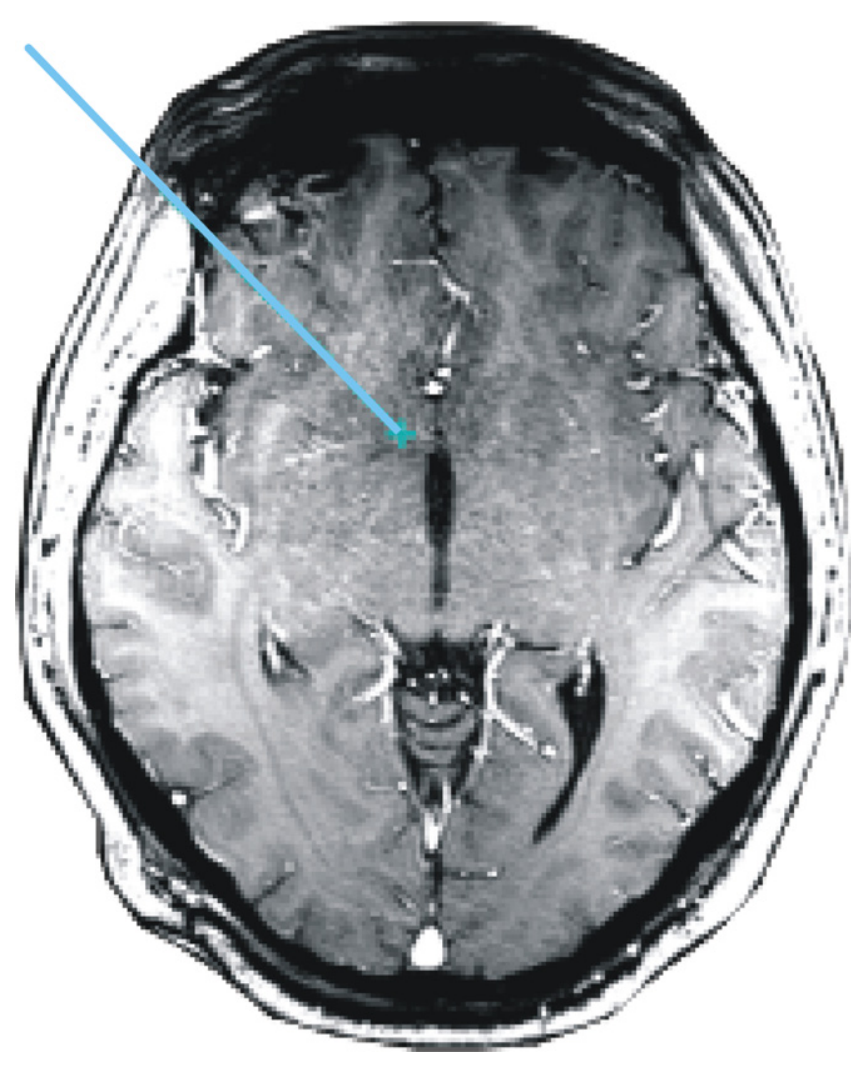

Figure 3. Location of the electrode. MRI scan showing the trajectory and tip of the DBS electrode within the Nucleus accumbens.

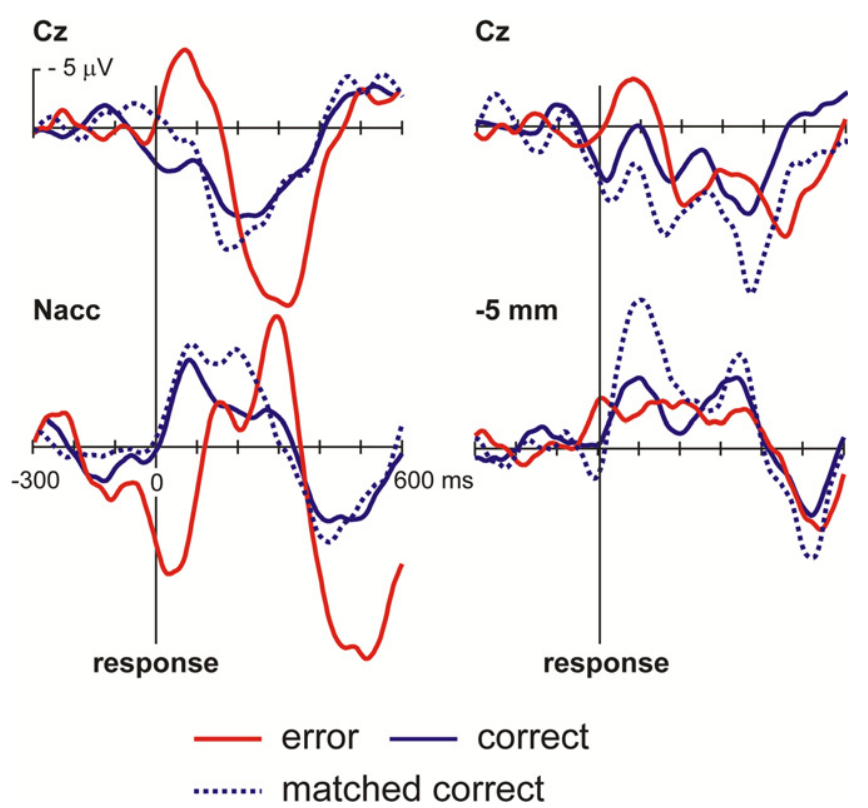

Figure 4. OCD patient. Intraoperative recordings. Shown are averaged ERPS from the surface $(\mathrm{Cz}-\mathrm{A1})$ and from the depth electrode within the Nacc or $5 \mathrm{~mm}$ above the target. Button press errors (25\% of trials) show a typical error-related negativity with a maximum at about $70 \mathrm{~ms}$ at $\mathrm{Cz}$. By contrast correct trials show a positive deflection. Clearly, activity from the Nacc proper but not from the region $5 \mathrm{~mm}$ above the target shows error-related modulations. To rule out the possibility that differences in brain responses are due to differences in reaction times in error and correct trials, correct trials matched to the error trials with regard to RT are also shown.

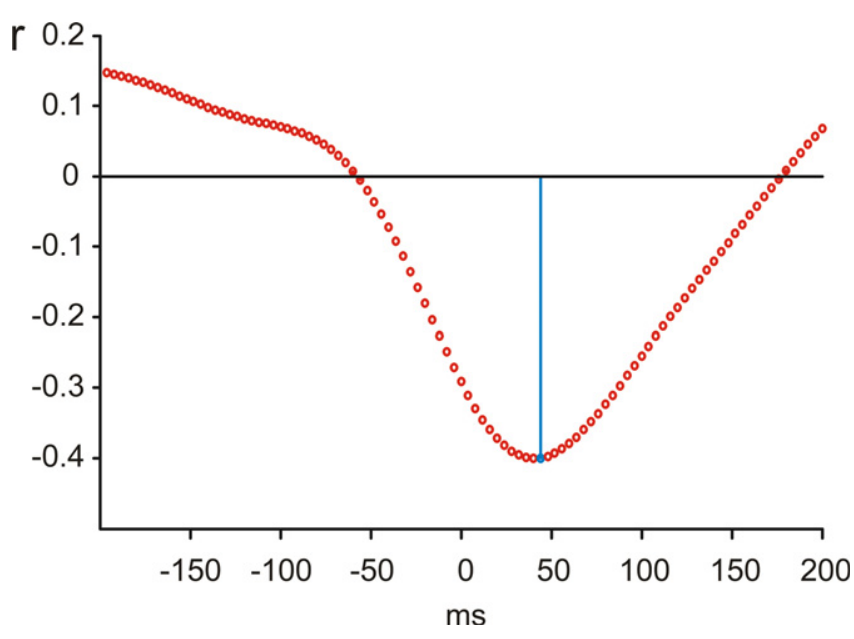

Figure 5. Cross-correlation of surface and Nacc single trial brain activity. Cross-correlation analysis indicates a delay of surface activity by about $40 \mathrm{~ms}$.
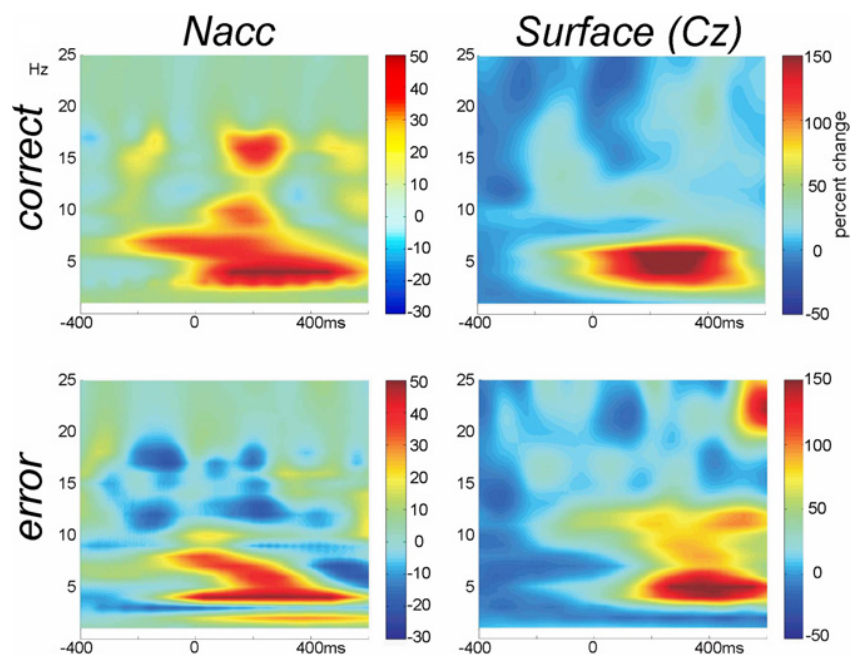

Figure 6. Time-frequency plots. Wavelet analysis of the recordings from the Nacc and the surface of the scalp (Cz-A1) recorded in parallel during the operation show performance related differences.

modulation, which was nearly absent in the recordings from a position $5 \mathrm{~mm}$ above the target.

In order to determine the temporal relationship between the activity at the surface and the Nacc activity, a cross-correlation analysis based on single trials was performed (Figure 5). This analysis revealed that the cortical error response was delayed relative to Nacc by about $40 \mathrm{~ms}$.

In Figure 6 we show the time-frequency plots. Depicted is the change in power (in percent relative to the baseline -300 to 0 with regard to stimulus onset).

\section{DISCUSSION}

In the current single patient study we present surface and intracerebral recordings from the Nucleus accumbens in a patient suffering from severe OCD. During the pretest an extraordinarily large ERN was obtained placing the patient at the $97^{\text {th }}$ percentile of our large sample of 83 normal participants. This fits well with previous investigations from our (Johannes et al., 2001, 2002) and other laboratories (Gehring et al., 2000; Hajcak and Simons, 2002; Santesso et al., 2006) which similarly have reported an 
increased amplitude of the ERN in OCD patients, patients with Tourette's syndrome with comorbid $\mathrm{OCD}$, and normal participants with obsessivecompulsive personality traits. These findings have been interpreted as reflecting increased action monitoring in OCD subjects, an interpretation which is also supported by a recent fMRI study that reported significantly greater error-related activation of the rostral ACC in OCD compared to healthy subjects (Fitzgerald et al., 2005). Moreover, activity in this region was positively correlated with symptom severity in the patients. It is interesting to speculate that these findings, and in particular the increased ERN amplitude in our patient, reflect excessive activity within the corticostriatal-pallidal-thalamic loop, which has been documented using different neuroimaging techniques such as positron-emission-tomography (PET; Perani et al., 1995; Rauch et al., 1994), single-photon emission computed tomography (SPECT; Machlin et al., 1991) or fMRI (Adler et al., 2000; Breiter et al., 1996). It has been speculated that enhanced activity of the error monitoring system might reflect a greater sensitivity to errors (Fitzgerald et al., 2005; Gehring et al., 2000; Johannes et al., 2001). Such enhanced sensitivity could manifest itself behaviourally by an increased tendency to slow responses after errors in order to avoid further slips on subsequent trials (posterror slowing, see Rabbitt, 1966, 1968) or in an enhanced error correction rate. We can not assess the latter, as error corrections were explicitly forbidden in the current experiment, but posterror slowing was $70.5 \mathrm{~ms}$ in our patient which compares with only $28 \mathrm{~ms}$ in the control subjects. Interestingly, the degree of posterror slowing has recently been shown to be positively correlated to activity in the medial prefrontal cortex (Klein et al., 2007). Taken together, this supports the notion of greater error sensitivity in our patient.

Importantly, and in accord with our hypothesis, we observed clear error-related modulations in time-domain averages of Nacc activity but not from a position $5 \mathrm{~mm}$ above the target region. This suggests that the Nacc is specifically involved in error processing and action monitoring and rules out the possibility that the activity is caused by volume conductance from distant sources (e.g., the ACC). The cross-correlation analysis further shows that the Nacc receives information about $40 \mathrm{~ms}$ prior to the medial frontal structure (ACC) that give rise to the ERN in surface recordings. In addition, performance-related modulations were also visible in waveletbased time-frequency analyses. In particular, the recordings from the Nacc show that error and correct trials were also differentiated in this analysis by a burst of activity in the beta-range $(14-17 \mathrm{~Hz}$ ) for the correct trials in addition to low frequency activity in the theta range. Using surface recordings of $\mathrm{EEG}$, we have recently been able to show a dichotomy of theta and beta oscillatory responses over the frontal midline, with the former being sensitive to negative and the latter correlated to positive events (MarcoPallares et al., 2008). It is interesting to speculate, whether a similar dichotomy could be demonstrated for the Nacc, but clearly recordings in a greater sample of patients are necessary to address this issue.

The Nacc receives inputs from the prefrontal cortex on the one hand and limbic structures such as the hippocampus and amygdala on the other (Groenewegen et al., 1999; Ikemoto and Panksepp, 1999). Moreover, its activity is modulated by dopaminergic neurons originating in the ventral tegmental area (Ikemoto and Panksepp, 1999; Voorn et al., 1986) which is a key area in incentive motivation (cf. Berridge, 2004). This circuitry allows for the integration of contextual information arising from hippocampus and emotional information coming from the amygdala with cognitive information supplied by the PFC in the selection of goal-directed behaviours (Grace, 2000), which is why the Nacc has been called a limbic-motor interface (Mogenson et al., 1980). With respect to the dopaminergic input into the Nacc, Goto and Grace (2005) found that tonic and phasic dopamine release has selective effects on hippocampal and cortical inputs via D1 and D2 receptors. They conclude that dopamine release "regulates the balance between limbic and cortical drive through activation and inactivation of DA receptor subtypes in the accumbens and this regulates goal-directed behaviour." What the current data, for the first time in humans, demonstrate is that the Nacc receives error signals that might be used to weigh the information coming from the different inputs in order to use this information for the adjustments of response strategies.

The current data also fits well with one of the more prominent theories that have been proposed for the generation of the ERN, i.e., the reinforcement learning theory of Holroyd and Coles (2002). This theory is based on the interaction of the mesencephalic dopamine system and a "generic" error-processing system located within the anterior cingulate cortex, the latter giving rise to the ERN. Specifically, the ERN is thought to be generated when a negative reinforcement learning signal is conveyed to the anterior cingulate cortex in the form of a decrease of dopaminergic activity coming from the mesencephalic dopamine system. We suggest that in addition to the anterior cingulate cortex, the Nacc receives a similar negative reinforcement signal that is used for the modification of its information processing.

The present investigation took advantage of the fact that the Nacc has been identified recently as a promising target for the treatment of $\mathrm{OCD}$ (Okun et al., 2007; Sturm et al., 2003; Tass et al., 2003; van Kuyck et al., 2007), Tourette syndrome (Kuhn et al., 2007), depression (Schlaepfer et al., 2008), and possibly addictive disorders (Kuhn et al., 2007). While our study was not intended to assess the clinical utility of the Nacc as a target of functional neurosurgery, we would like to briefly consider some clinical aspects of this therapy. In a recent review, summarizing animal and patient studies, van Kuyck et al. (2007) demonstrated effects of electrical stimulation of the Nacc on a variety of behaviours including reward processing, goal-directed activity, fight-or-flight response patterns, degree of exploratory behaviour and food intake. The possible value of the Nacc as a target structure has been underscored by recent modelling work linking this structure in the etiology of craving and obsessions (Redish and Johnson, 2007). The influence of DBS of a neighbouring region, that is, the ventral anterior internal capsule/ventral striatum, on regional cerebral blood flow has recently been investigated using PET in an on/off design in a small group of OCD patients (Rauch et al., 2006). During stimulation a significant activation of orbitofrontal cortex, anterior cingulate cortex, striatum, globus pallidus, and thalamus was found: These are brain regions that have been implicated in $0 C D$ on the one hand and, at least with regard to the anterior cingulate cortex, action monitoring on the other hand. Whether or not stimulation of the Nacc or the ventral striatum resets action monitoring circuits to a more normal level in OCD remains a topic for future investigation.

\section{CONCLUSION}

The invasive electrophysiological recordings from the Nacc suggest that this structure is involved in action monitoring. Because of its strategic location, Nacc may use the error signal to adjust the weight of limbic and prefrontal inputs on control structures that optimize goal-directed behaviour.

\section{CONFLICT OF INTEREST STATEMENT}

The authors declare that the research was conducted in the absence of any commercial or financial relationships that could be construed as a potential conflict of interest.

\section{ACKNOWLEDGEMENTS}

We thank Antoni Rodriguez-Fornells, Nadine Strien and Jordi Riba for contributing the data of the healthy participants.

This work was supported by grants from the BMBF to TFM, HJH and VS, and grants from the DFG to TFM and $\mathrm{HJH}$.

\section{REFERENCES}

Adler, C. M., Donough-Ryan, P., Sax, K. W., Holland, S. K., Arndt, S., and Strakowski, S. M. (2000). fMRI of neuronal activation with symptom provocation in unmedicated patients with obsessive compulsive disorder. J. Psychiatr. Res. 34, 317-324.

Barto, A. G. (1995). Adaptive critics and the basal ganglia. In Models of Information Processing in the Basal Ganglia, J. Houk, J. Davis and D. Beiser, eds (Cambridge, MA, MIT Press), pp. 215-232. 
Berridge, K. C. (2004). Motivation concepts in behavioral neuroscience. Physiol. Behav. 81, 179-209.

Botvinick, M. M., Braver, T. S., Barch, D. M., Carter, C. S., and Cohen, J. D. (2001). Conflict monitoring and cognitive control. Psychol. Rev. 108, 624-652.

Breiter, H. C., Rauch, S. L., Kwong, K. K., Baker, J. R., Weisskoff, R. M., Kennedy, D. N. Kendrick, A. D., Davis, T. L., Jiang, A., Cohen, M. S., Stern, C. E., Belliveau, J. W. Baer, L., O'Sullivan, R. L., Savage, C. R., Jenike, M. A., and Rosen, B. R. (1996) Functional magnetic resonance imaging of symptom provocation in obsessivecompulsive disorder. Arch. Gen. Psychiatry 53, 595-606.

Carter, C. S., Braver, T. S., Barch, D. M., Botvinick, M. M., Noll, D., and Cohen, J. D. (1998) Anterior cingulate cortex, error detection, and the online monitoring of performance. Science 280, 747-749.

Chatrian, G. E., Lettich, E., and Nelson, P. L. (1985). Ten percent electrode system for topographic studies of spontaneous and evoked EEG activity. Am. J. EEG Technol. $25,83-92$.

Chatrian, G. E., Lettich, E., and Nelson, P. L. (1988). Modified nomenclature for the ' $10 \%$ ' electrode system. J. Clin. Neurophysiol. 5, 183-186

Crawford, J. R., and Howell, D. C. (1998). Comparing an individual's test score against norms derived from small samples. Clin. Neuropsychol. 12, 482-486.

Eriksen, B. A., and Eriksen, C. W. (1974). Effects of noise letters upon the identification of target letters in a non-search task. Percept. Psychophys. 16, 143-149.

Falkenstein, M., Hohnsbein, J., Hoormann, J., and Blanke, L. (1990). Effects of errors in choice reaction task on the ERP under focused and divided attention. In Psychophysiological Brain Research, C. H. M. Brunia, A. W. K. Gaillard and A. Kok, eds (Tilburg, University Press), pp. 192-195.

Fassbender, C., Murphy, K., Foxe, J. J., Wylie, G. R., Javitt, D. C., Robertson, I. H., and Garavan, H. (2004). A topography of executive functions and their interactions revealed by functional magnetic resonance imaging. Brain Res. Cogn. Brain Res. 20,132-143.

Fiehler, K., Ullsperger, M., and von Cramon, D. Y. (2005). Electrophysiological correlates of error correction. Psychophysiology 42, 72-82.

Fitzgerald, K. D., Welsh, R. C., Gehring, W. J., Abelson, J. L., Himle, J. A., Liberzon, I., and Taylor, S. F. (2005). Error-related hyperactivity of the anterior cingulate cortex in obsessive-compulsive disorder. Biol. Psychiatry 57, 287-294.

Gehring, W. J., Goss, B., Coles, M. G. H., Meyer, D. E., and Donchin, E. (1993). A neural system for error-detection and compensation. Psychol. Sci. 4, 385-390.

Gehring, W. J., Himle, J., and Nisenson, L. G. (2000). Action-monitoring dysfunction in obsessive-compulsive disorder. Psychol. Sci. 11, 1-6.

Goto, Y., and Grace, A. A. (2005). Dopaminergic modulation of limbic and cortical drive of nucleus accumbens in goal-directed behavior. Nat. Neurosci. 8, 805-812.

Grace, A. A. (2000). Gating of information flow within the limbic system and the pathophysiology of schizophrenia. Brain Res. Brain Res. Rev. 31, 330-341.

Groenewegen, H. J., Wright, C. I., Beijer, A. V., and Voorn, P. (1999). Convergence and segregation of ventral striatal inputs and outputs. Ann. N. Y. Acad. Sci. 877, 49-63.

Hajcak, G., and Simons, R. F. (2002). Error-related brain activity in obsessive-compulsive undergraduates. Psychiatry Res. 110, 63-72.

Helmstaedter, C., Lendt, M., and Lux, S. (2001). Verbaler Lern- und Merkfähigkeitstest (VLMT). Göttingen, Hogrefe.

Hester, R., Fassbender, C., and Garavan, H. (2004). Individual differences in error processing: a review and reanalysis of three event-related fMRI studies using the G0/NOG0 task. Cereb. Cortex 14, 986-994.

Holroyd, C. B., and Coles, M. G. H. (2002). The neural basis of human error processing reinforcement learning, dopamine, and the error-related negativity. Psychol. Rev. 109, 679-709.

Houk, J., Adams, J. L., and Barto, A. G. (1995). A model of how the basal ganglia generate and use neural signals that predict reinforcement. In Models of Information Processing in the Basal Ganglia, J. Houk, J. Davis and D. Beiser, eds (Cambridge, MA MIT Press), pp. 249-270.

Ikemoto, S., and Panksepp, J. (1999). The role of nucleus accumbens dopamine in motivated behavior: a unifying interpretation with special reference to reward-seeking Braiin Res. Rev. 31, 6-41.

Johannes, S., Wieringa, B. M., Nager, W., Muller-Vahl, K. R., Dengler, R., and Munte, T. F (2002). Excessive action monitoring in Tourette syndrome. J. Neurol. 249, 961-966.

Johannes, S., Wieringa, B. M., Nager, W., Rada, D., Dengler, R., Emrich, H. M., Munte, T. F. and Dietrich, D. E. (2001). Discrepant target detection and action monitoring in obsessive-compulsive disorder. Psychiatry Res. 108, 101-110.

Kelley, A. E., and Berridge, K. C. (2002). The neuroscience of natural rewards: relevance to addictive drugs. J. Neurosci. 22, 3306-3311.

Klein, T. A., Endrass, T., Kathmann, N., Neumann, J., von Cramon, D. Y., and Ullsperger, M. (2007). Neural correlates of error awareness. Neuroimage 34, 1774-1781.

Kuhn, J., Lenartz, D., Mai, J. K., Huff, W., Lee, S. H., Koulousakis, A., Klosterkoetter, J., and Sturm, V. (2007). Deep brain stimulation of the nucleus accumbens and the internal capsule in therapeutically refractory Tourette-syndrome. J. Neurol. 254, 963-965.

Lezak, M. D. (1995). Neuropsychological Assessment, 3rd Edn. New York, NY, Oxford University Press.

Luu, P., and Tucker, D. M. (2001). Regulating action: alternating activation of midline frontal and motor cortical networks. Clin. Neurophysiol. 112, 1295-1306.

Machlin, S. R., Harris, G. J., Pearlson, G. D., Hoehn-Saric, R., Jeffery, P., and Camargo, E. E. (1991). Elevated medial-frontal cerebral blood flow in obsessive-compulsive patients: a SPECT study. Am. J. Psychiatry 148, 1240-1242.
Marco-Pallares, J., Cucurell, D., Cunillera, T., Garcia, R., ndres-Pueyo, A., Munte, T. F., and Rodriguez-Fornells, A. (2008). Human oscillatory activity associated to reward processing in a gambling task. Neuropsychologia 46, 241-248.

Mogenson, G. J., Jones, D. L., and Yim, C. Y. (1980). From motivation to action: functional interface between the limbic system and the motor system. Prog. Neurobiol. 14 69-97.

Montague, P. R., Dayan, P., and Sejnowski, T. J. (1996). A framework for mesencephalic dopamine systems based on predictive Hebbian learning. J. Neurosci. 16 , 1936-1947.

Nieuwenhuis, S., Nielen, M. M., Mol, N., Hajcak, G., and Veltman, D. J. (2005). Performance monitoring in obsessive-compulsive disorder. Psychiatry Res. 134, 111-122.

Okun, M. S., Mann, G., Foote, K. D., Shapira, N. A., Bowers, D., Springer, U., Knight, W., Martin, P., and Goodman, W. K. (2007). Deep brain stimulation in the internal capsule and nucleus accumbens region: responses observed during active and sham programming. J. Neurol. Neurosurg. Psychiatr. 78, 310-314.

Perani, D., Colombo, C., Bressi, S., Bonfanti, A., Grassi, F., Scarone, S., Bellodi, L., Smeraldi, E., and Fazio, F. (1995). [18F]FDG PET study in obsessive-compulsive disorder. A clinical/metabolic correlation study after treatment. Br. J. Psychiatry 166, 244-250.

Rabbitt, P. (2002). Consciousness is slower than you think. Q. J. Exp. Psychol. A 55, $1081-1092$

Rabbitt, P. M. (1966). Errors and error correction in choice-response tasks. J. Exp. Psychol. 71, 264-272.

Rabbitt, P. M. (1968). Three kinds of error-signalling responses in a serial choice task. Q. J. Exp. Psychol. 20, 179-188.

Rauch, S. L., Dougherty, D. D., Malone, D., Rezai, A., Friehs, G., Fischman, A. J.,Alpert, N. M., Haber, S. N., Stypulkowski, P. H., Rise, M. T., Rasmussen, S. A., and Greenberg, B. D. (2006). A functional neuroimaging investigation of deep brain stimulation in patients with obsessive-compulsive disorder. J. Neurosurg. 104, 558-565.

Rauch, S. L., Jenike, M. A., Alpert, N. M., Baer, L., Breiter, H. C., Savage, C. R., and Fischman, A. J. (1994). Regional cerebral blood flow measured during symptom provocation in obsessive-compulsive disorder using oxygen 15-labeled carbon dioxide and positron emission tomography. Arch. Gen. Psychiatry 51, 62-70.

Redish, A. D., and Johnson, A. (2007). A computational model of craving and obsession. Ann. N. Y. Acad. Sci. 1104, 324-339.

Riba, J., Rodriguez-Fornells, A., Munte, T. F., and Barbanoj, M. J. (2005). A neurophysiological study of the detrimental effects of alprazolam on human action monitoring. Brain Res. Cogn. Brain Res. 25, 554-565.

Rodriguez-Fornells, A., Kurzbuch, A. R., and Munte, T. F. (2002). Time course of error detection and correction in humans: neurophysiological evidence. J. Neurosci. 22, 9990-9996

Rollnik, J. D., Schroder, C., Rodriguez-Fornells, A., Kurzbuch, A. R., Dauper, J., Moller, J., and Munte, T. F. (2004). Functional lesions and human action monitoring: combining repetitive transcranial magnetic stimulation and event-related brain potentials. Clin. Neurophysiol. 115, 145-153.

Santesso, D. L., Segalowitz, S. J., and Schmidt, L. A. (2006). Error-related electrocortical responses are enhanced in children with obsessive-compulsive behaviors. Dev. Neuropsychol. 29, 431-445.

Schlaepfer, T. E., Cohen, M. X., Frick, C., Kosel, M., Brodesser, D., Axmacher, N., Joe, A. Y., Kreft, M., Lenartz, D., and Sturm, V. (2008). Deep brain stimulation to reward circuitry alleviates anhedonia in refractory major depression. Neuropsychopharmacology 33 368-377.

Schultz, W. (2006). Behavioral theories and the neurophysiology of reward. Annu. Rev. Psychol. 57, 87-115.

Sturm, V., Lenartz, D., Koulousakis, A., Treuer, H., Herholz, K., Klein, J. C., and Klosterkotter, J. (2003). The nucleus accumbens: a target for deep brain stimulation in obsessive-compulsive- and anxiety-disorders. J. Chem. Neuroanat. 26, 293-299.

Tallon-Baudry, C., Bertrand, 0., Delpuech, C., and Pernier, J. (1997). Oscillatory gammaband $(30-70 \mathrm{~Hz})$ activity induced by a visual search task in humans. J. Neurosci. $17,722-734$.

Tass, P. A., Klosterkotter, J., Schneider, F., Lenartz, D., Koulousakis, A., and Sturm, V. (2003). Obsessive-compulsive disorder: development of demand-controlled deep brain stimulation with methods from stochastic phase resetting. Neuropsychopharmacology 28, S27-S34.

Ullsperger, M., and von Cramon, D. Y. (2001). Subprocesses of performance monitoring: a dissociation of error processing and response competition revealed by eventrelated fMRI and ERPs. Neuroimage 14, 1387-1401.

Ullsperger, M., and von Cramon, D. Y. (2003). Error monitoring using external feedback: specific roles of the habenular complex, the reward system, and the cingulate motor area revealed by functional magnetic resonance imaging. J. Neurosci. 23, 4308-4314.

van Kuyck, K., Gabriels, L., Cosyns, P., Arckens, L., Sturm, V., Rasmussen, S., and Nuttin, B. (2007). Behavioural and physiological effects of electrical stimulation in the nucleus accumbens: a review. Acta Neurochir. 97, 375-391.

Voorn, P., Jorritsma-Byham, B., Van, D. C., and Buijs, R. M. (1986). The dopaminergic innervation of the ventral striatum in the rat: a light- and electron-microscopical study with antibodies against dopamine. J. Comp. Neurol. 251, 84-99.

Zimmermann, P., and Fimm, B. (2002). Testbatterie zur Aufmerksamkeitsprüfung (TAP). Version 1.7. Freiburg, Psytest. 